\title{
Hallux valgus leve y moderado; tratamiento mediante cirugía de invasión mínima y su seguimiento a 2 años
}

\author{
Hallux valgus mild and moderate; treatment by minimally \\ invasive surgery and its follow-up to 2 years
}

King-Martínez AC, ${ }^{*}$, Estevez-Jaramillo MA, ${ }^{\S}$ King-Hayata MA, ${ }^{\circledR}$ Martínez-de Anda MC, ${ }^{\ddagger}$ Cuellar-Avaroma A

Médica Sur.

RESUMEN. Introducción: El hallux valgus es una de las patologías más frecuentes y complejas en la ortopedia. Afecta a los tejidos blandos y óseos del primer dedo. Se encuentran descritas múltiples técnicas para su corrección, todas con un último fin: lograr restaurar el ángulo fisiológico del primer dedo mediante una osteotomía estable y con el menor dolor postquirúrgico posible. Las técnicas de invasión mínima han venido ganando adeptos desde los años 2000 como una alternativa para el tratamiento de esta patología. Material y métodos: Se incluyeron pacientes con diagnóstico de hallux valgus leve o moderado en los que se realizó un procedimiento mínimamente invasivo para corregir la deformidad del primer dedo del pie. Se realizó una osteotomía distal de Reverdin/Isham en el primer metatarsiano y una osteotomía de Akin en la falange proximal, una liberación capsular lateral y una tenotomía abductora. Estos casos fueron seguidos durante 24 meses después de su cirugía. Resultado: Nuestros pacientes presentaron una adecuada corrección de la sintomatología dolorosa, retorno a la movilidad articular prequirúrgica y una adecuada satisfacción personal con $87.3 \%$ de buenos resultados. En-
ABSTRACT. Introduction: The hallux valgus is a very frequent and complex orthopedic pathology. It involves the bone and soft tissue structures of the first toe. There are multiple techniques described for the alignment of the first toe. All technics aim to restore the normal angulation of the toe while using a stable osteotomy through a painless surgical procedure. The minimal invasive techniques have been growing in acceptance since the year 2000, as a viable surgical alternative to treat this pathology. Material and methods: We included patients with a diagnostic of mild or moderate hallux valgus on whom a minimal invasive procedure was performed to correct the deformity of the first toe. We performed a distal Reverdin/Isham osteotomy on the first metatarsal and an akin osteotomy in the proximal phalanx, a lateral capsular release and a abductor tenotomy. These patients were followed for 24 months after their surgery. Results: Our patients had an adequate correction angular correction, for a distal osteotomy. They had a good pain control, with an adequate mobility in the postoperative period. The patients presented an adequate personal satisfaction, $87 \%$ of them had good results. We found an

\section{Nivel de evidencia: IV}

* Ortopedia y Traumatología Médico adscrito Hospital General «Dr. Manuel Gea González».

* Ortopedia y Traumatología, Médico adscrito a la Clínica de Pie y Tobillo, Médica Sur.

§ Salud Pública y Global.

` Ortopedia y Traumatología Coordinador de la Clínica de Pie y Tobillo, Médica Sur.

Correspondencia:

Dra. Ana Cristina King-Martínez

Puente de Piedra Núm. 150, Torre 2, Consultorio 118,

Col. Toriello Guerra, C.P. 14050 ,

Alcaldía Tlalpan, Ciudad de México.

Tel: 55 5666-0808 y 55 5424-3165

E-mail: anacristinaking@kingortopedia.com

Recibido: 15-12-2020. Aceptado: 01-08-2021.

Citar como: King-Martínez AC, Estevez-Jaramillo MA, King-Hayata MA, Martínez-de Anda MC, Cuellar-Avaroma A. Hallux valgus leve y moderado; tratamiento mediante cirugía de invasión mínima y su seguimiento a 2 años. Acta Ortop Mex. 2021; 35(4): 305-310. https://dx.doi.org/10.35366/103308 
contramos una mejoría importante en la escala de Kitaoka. Tenemos una adecuada corrección angular para una osteotomía distal. Conclusiones: Las técnicas de invasión mínima para la corrección de hallux valgus moderado y leve son una adecuada herramienta. Nuestros pacientes se encuentran satisfechos con los resultados estéticos y funcionales.

Palabras clave: Hallux valgus, tratamiento, mínima invasión, resultados.

\section{Introducción}

El hallux valgus (HV) es una de las patologías más frecuentes y complejas en la ortopedia. Afecta tanto a los tejidos blandos como óseos del primer dedo. Es una deformidad causada por una subluxación progresiva, en valgo de la articulación metatarso-falángica (MTF) del primer dedo y una desviación en varo del primer metatarsiano. Se presenta más comúnmente en pacientes de sexo femenino. Se considera una patología multifactorial. Estas deformidades se ven comúnmente acompañadas de deformidades o síntomas de los dedos restantes del pie. Al desviarse el primer dedo y aumentar el dolor, el paciente tiende a cargar mayor porcentaje del peso en las cabezas de los metatarsianos menores, aumentando así la posibilidad de presentar metatarsalgias en dichos dedos, así como hiperqueratosis o hasta fracturas por sobrecarga (estrés). Los dedos menores se deforman, causando a su vez dolor y limitando el uso de calzado considerado normal por el paciente. ${ }^{1,2}$

Existen múltiples técnicas quirúrgicas encaminadas a resolver esta deformidad, todas tratando de disminuir el dolor del paciente al caminar y calzar. Estos procedimientos pueden ser agrupados en: plastías de tejidos blandos, osteotomías correctoras, artroplastías de resección y artrodesis. ${ }^{3}$ important and statistically significant improvement in the Kitaoka scale. Conclusion: The minimal invasive technics for the correction of mild or moderated hallux valgus are a good alternative. Our patients are satisfied with the functional, and cosmetic results.

Keywords: Hallux valgus, treatment, minimal invasive surgery, results.

Las técnicas de cirugía de incisión mínima (CIM) también conocida como MIS por sus siglas en inglés (Figura 1), cirugía percutánea o cirugía de invasión mínima que utilizamos actualmente fueron descritas por el Dr. Stephen Isham a principios de los años noventa y popularizadas por el Dr. Mariano del Prado a inicios de los años 2000..$^{4,5}$ Estas técnicas de CIM permiten tanto la manipulación de tejidos óseos como blandos para lograr la corrección del HV.

El hallux valgus puede ser clasificado como leve, moderado o severo de acuerdo al ángulo intermetatarsal (IM) entre el primero y segundo metatarsiano. ${ }^{6,7}$

Los objetivos del presente estudio fueron dar respuesta a las siguientes preguntas: ¿las técnicas de CIM son un tratamiento adecuado para el HV leve y moderado?, ¿se logra un control del dolor y una mejoría funcional de nuestros pacientes?, ¿logramos una adecuada corrección angular con estas técnicas quirúrgicas?

\section{Material y métodos}

Nuestra serie de casos se basa en los 36 pacientes (63 pies) que fueron atendidos por nuestro grupo de cirujanos durante el período de 12 meses, con un seguimiento mínimo de 24 meses. A todos los pacientes se les realizó al menos:
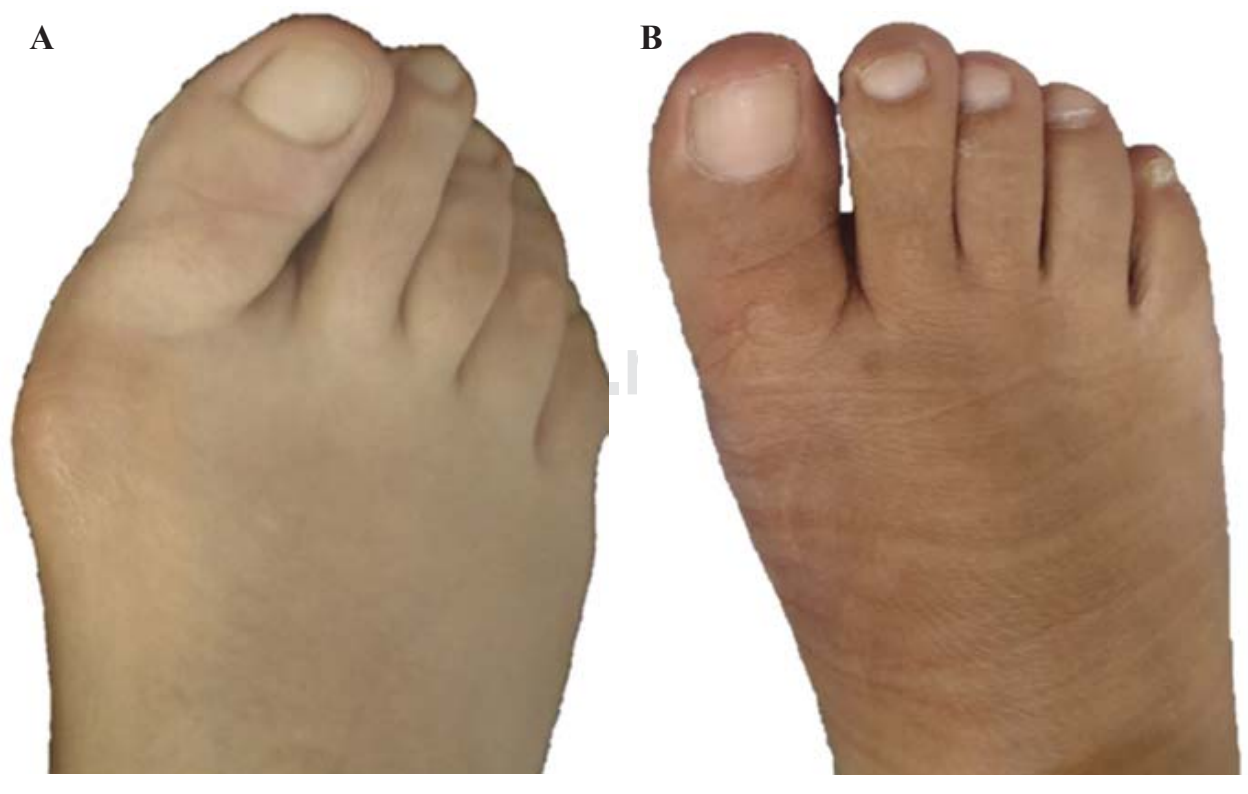

Figura 1:

A) Fotografía clínica prequirúrgica. B) Postquirúrgica a los dos meses. 
Tabla 1: Características de edad y género de los pacientes incluidos en el estudio. $N=36,63$ pies.

\begin{tabular}{|cc|}
\hline & $\mathrm{n}(\%)$ \\
\hline Edad (años) & \\
$\leq 49$ & $15(41.7)$ \\
$>49$ & $21(58.3)$ \\
Género (63 pies) & \\
Femenino & 33,58 pies $(92.1)$ \\
Masculino & 3,5 pies $(7.9)$ \\
\hline
\end{tabular}

Tabla 2: Patologías de pie asociadas al hallux valgus. $\mathrm{N}=63$ pies.

$\begin{array}{lc}\text { Patologías de pie asociadas } & \mathrm{n}(\%) \\ \text { Ninguna } & 20(31.7) \\ \text { Pie plano transverso } & 8(12.7) \\ \text { Metatarsalgia } & 15(23.8) \\ \text { Dedos en garra } & 15(23.8) \\ \text { Quinto supraducto } & 2(3.2) \\ \text { Juanetillo de sastre } & 1(1.6) \\ \text { Fascitis plantar } & 1(1.6) \\ \text { Haglund } & 1(1.6)\end{array}$

1. Exostosectomía metafisaria distal del primer metatarsiano.

2. Osteotomía distal del primer metatarsiano tipo ReverdinIsham (oblicua con cuña interna).

3. Osteotomía de la base de la falange proximal del primer dedo tipo Akin en cuña medial.

4. Capsulotomía inferolateral de la articulación metatarsofalángica del primer dedo.

5. Tenotomía del tendón abductor del primer dedo, a nivel de la articulación metatarso-falángica.

De nuestros pacientes, nueve (25\%) fueron operados de manera unilateral y 27 (75\%) de manera bilateral. Todos fueron seguidos por al menos 24 meses.

Las características de edad y género de nuestros pacientes se muestran en la Tabla 1.

Algunos de nuestros pacientes presentaron patologías de pie asociadas como se muestra en la Tabla 2.

Todos fueron valorados pre- y postquirúrgicamente, fueron intervenidos quirúrgicamente por los cirujanos de nuestro grupo. Dentro de la consulta prequirúrgica se realizó una valoración de la escala funcional validada de la AOFAS, la escala visual análoga del dolor y la movilidad de la primera metatarso-falángica.

\section{Resultados}

Los pacientes fueron mayormente femeninos con más de $92 \%$ de nuestra población con una edad promedio de 49 años. Sólo $31.7 \%$ de nuestros pacientes no tenían una patología asociada al hallux valgus. Las patologías más frecuentemente asociadas son las metatarsalgias centrales y los dedos en garra ambas con $23.8 \%$.
En cuanto a la movilización del dedo, la flexión dorsal cambió de $52.71^{\circ}$ a $54.29^{\circ}$ a los dos años de seguimiento, diferencia que no es estadísticamente significativa. La flexión plantar pasó de $11.11^{\circ}$ a $11.03^{\circ}$ también sin diferencia significativa. Estos valores reflejan que la movilidad del primer dedo de nuestros pacientes tras el procedimiento se mantuvo al menos igual que previo al procedimiento quirúrgico (Figura 2).

En cuanto a las mediciones radiográficas, estas se toman siempre en proyecciones dorsoplantar y lateral que se toman con los pacientes de pie y con apoyo (Figura 3). Los valores de las mediciones tanto prequirúrgicas como postquirúrgicas se pueden ver en la Figura 4. Nuestros resultados reflejan una disminución en promedio de $2^{\circ}$ del ángulo intermetatarsal. Estos valores reflejan una variación de los ángulos consistente con las correcciones logradas en otros estudios mediante el uso de osteotomías distales. ${ }^{8,9}$

En cuanto a la escala visual análoga del dolor (Figura 5), logramos pasar de más de 8 puntos de dolor a 1.49 puntos en promedio.

A todos nuestros pacientes les aplicamos en el pre- y postquirúrgico la escala de Kitaoka ahora conocida también como escala AOFAS, la cual fue desarrollada en 1994 y es una de las más utilizadas en la actualidad. Con esta escala podemos valorar de forma objetiva y ordenada la mejoría de nuestros pacientes. ${ }^{8}$ Esta escala, que consta de 100 puntos, combina la alineación de muestro primer metatarsiano (10 puntos), el dolor que presenta nuestro paciente (40 puntos) y la funcionalidad al evaluar las actividades que puede realizar (50 puntos). En nuestros pacientes, la evaluación funcional fue de 49.24 puntos prequirúrgicos a 94.32 puntos en el postquirúrgico. Con una diferencia media de 45.08 puntos, lo cual es estadísticamente significativo (Figura 6).

En todos medimos la satisfacción personal que presentaban tras el procedimiento quirúrgico. Este valor fue interrogado por otra persona diferente al médico tratante para

Mediciones clínicas pre- y postoperatorias

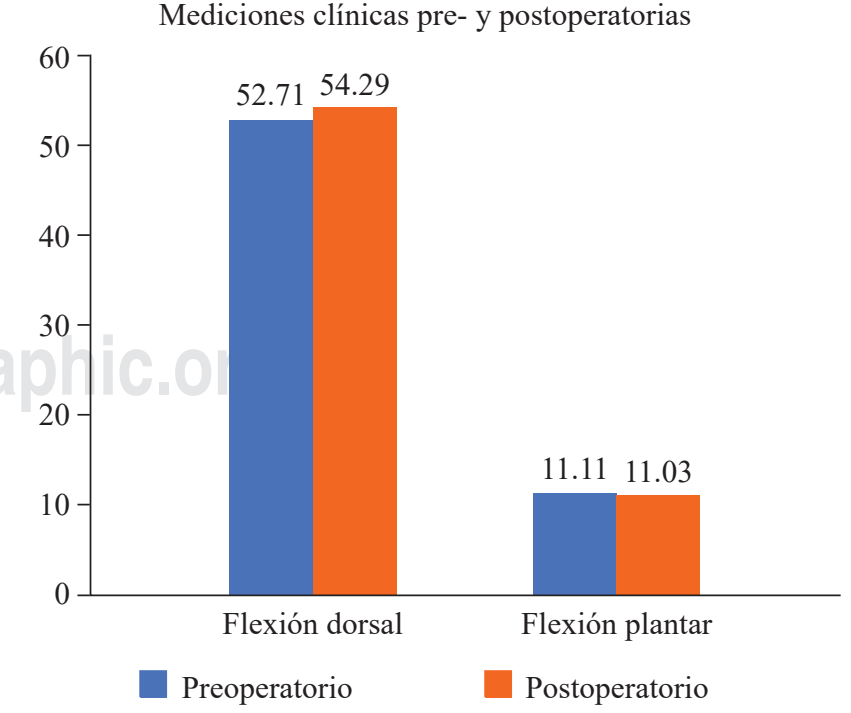

Figura 2: Comparación de medición de movimiento del primer dedo. 


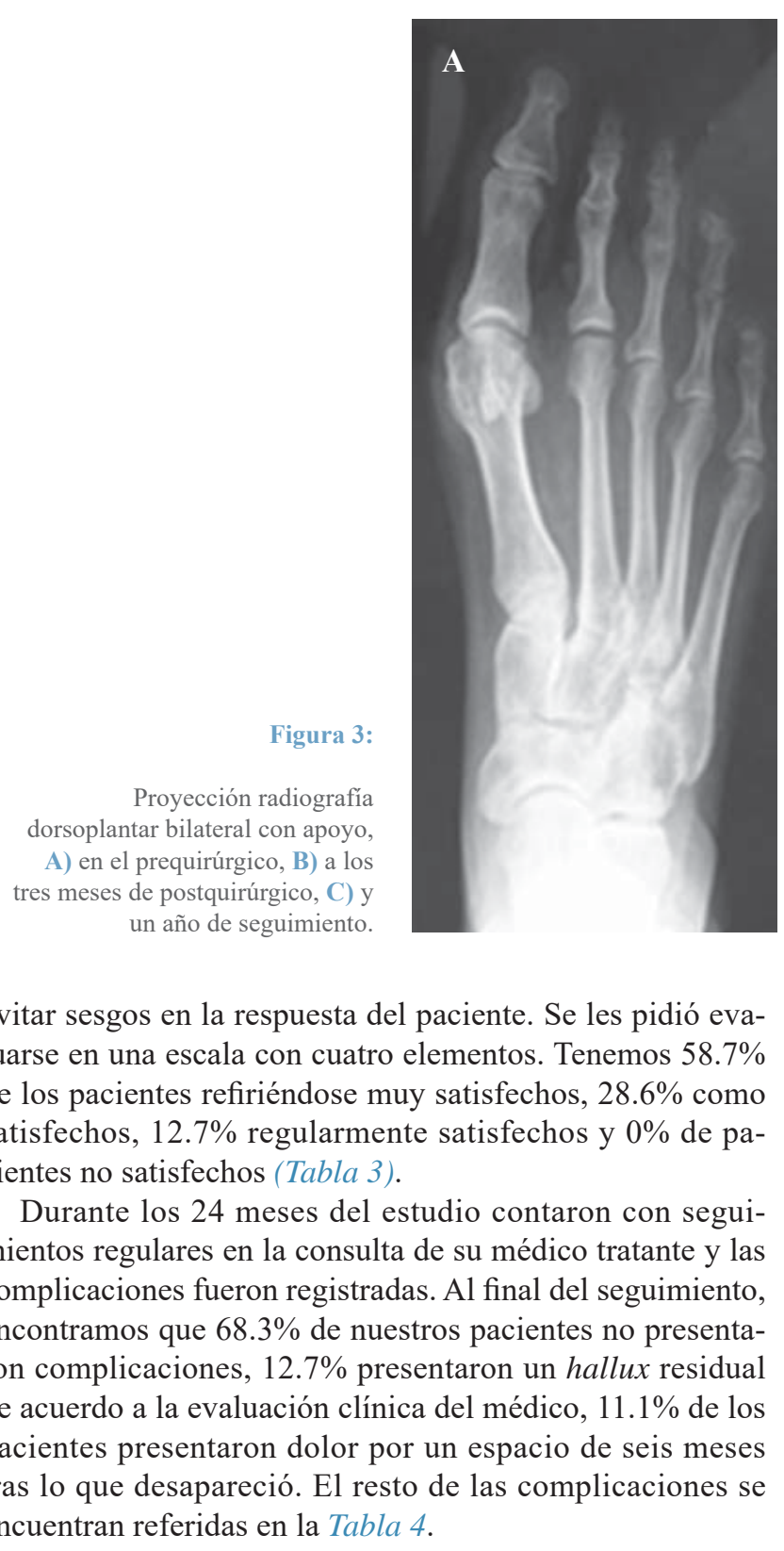

\section{Discusión}

Pese a que los cambios en la movilidad del primer dedo no tienen una diferencia significativa, sí nos demuestran que después del procedimiento quirúrgico correctivo y después del tiempo de seguimiento de nuestros pacientes el movimiento de la articulación del primer dedo, que nos permite realizar el despegue durante la marcha, permanece al menos en el mismo nivel que antes del procedimiento. Con base en estos resultados, podemos ofrecerle a nuestros casos que no habrá rigidez articular del primer dedo después del procedimiento quirúrgico.

En cuanto a las correcciones angulares, podemos observar que en esta investigación realizamos una osteotomía distal del primer metatarsiano y nuestro resultado fue la disminución de un promedio de $2^{\circ}$ del ángulo intermetatarsal. Este resultado es consistente en lo publicado por otros
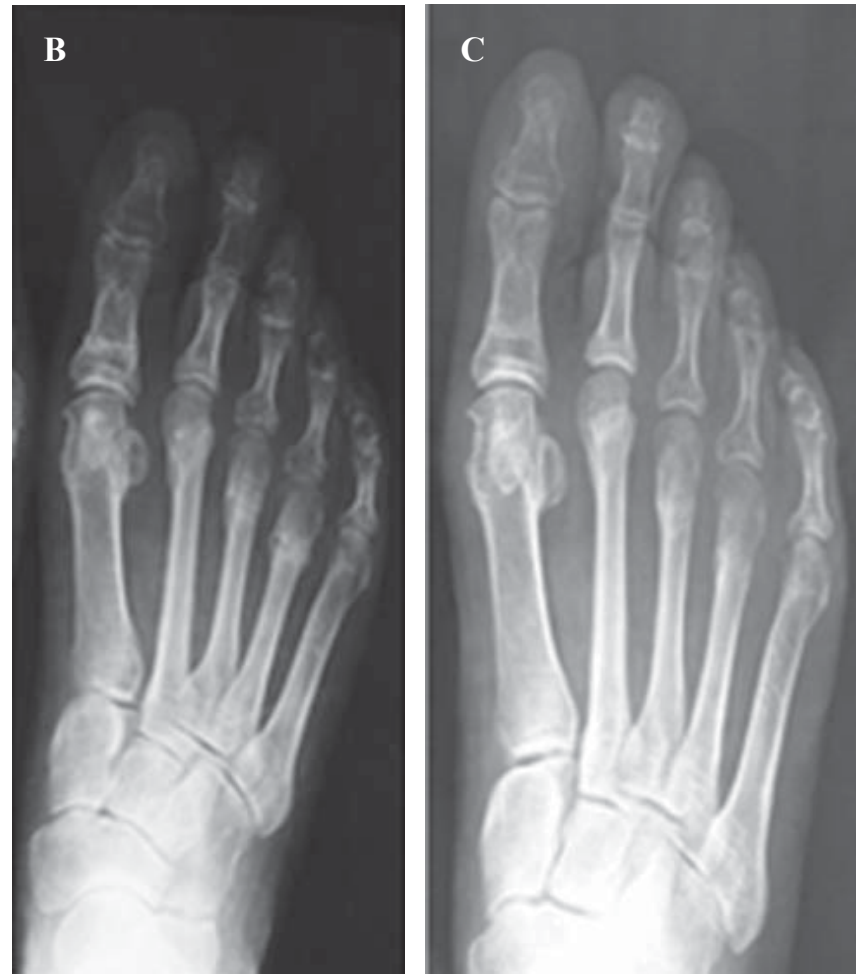

Mediciones radiológicas pre- y postoperatorias

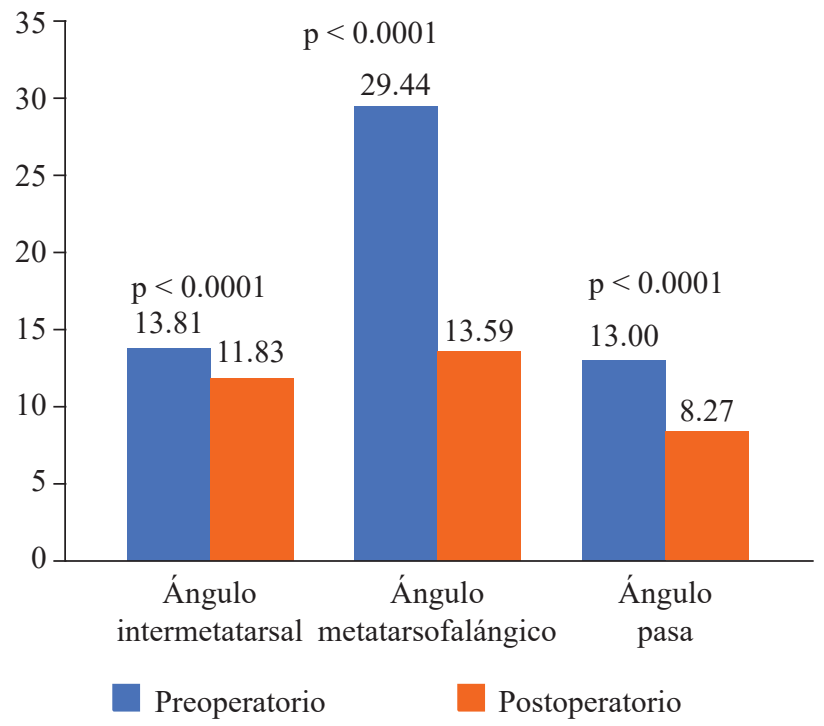

Figura 4: Comparación de mediciones radiológicas pre- y postoperatorias.

autores. ${ }^{10,11,12,13,14}$ En cuanto al ángulo PASA, encontramos una disminución de $4.7^{\circ}$ en promedio, esto se logra debido a que nuestra osteotomía distal es una cuña de cierre medial que logra una corrección en la inclinación de la superficie articular distal del primer metatarsiano. ${ }^{15}$

En cuanto al ángulo metatarso-falángico, con nuestro procedimiento logramos una importante corrección del mismo pudiendo lograr una corrección promedio de $15^{\circ}$. 
En la Figura 4 podemos encontrar la muy importante diferencia en cuanto al dolor que refiere nuestro paciente de las condiciones prequirúrgicas a las postquirúrgicas a dos años de su intervención, así este procedimiento quirúrgico nos ayuda a aliviar el dolor que presentan al lograr las correcciones de las deformidades de esta patología. Podemos además conocer que esta mejoría perdura en el tiempo, al menos durante el tiempo de seguimiento de nuestros casos.

Encontramos complicaciones menores en $31.8 \%$ de los pacientes que se asemeja a lo publicado en la literatura. ${ }^{16,17}$ Una de las debilidades del estudio es el número reducido de casos que lo conforman.

\section{Conclusión}

El tratamiento del hallux valgus leve y moderado con cirugía de invasión mínima es una opción que ofrece una adecuada corrección angular, $31 \%$ de complicaciones en

Escala visual análoga

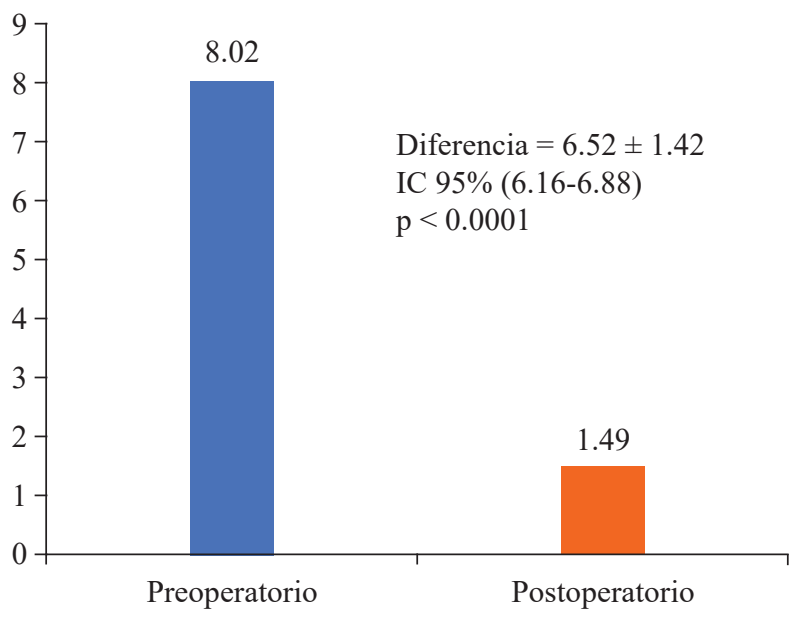

Figura 5: Evaluación del dolor.



Escala de Kitaoka

Figura 6: Evaluación funcional.
Tabla 3: Satisfacción de los pacientes sometidos

a cirugía percutánea entre Enero y Diciembre del 2008 con seguimiento a 24 meses.

\begin{tabular}{|lrc|}
\hline Grado de satisfacción & $\%$ & Acumulativo, \% \\
\hline Muy satisfecho & 58.7 & 58.7 \\
Satisfecho & 28.6 & 87.3 \\
Regularmente satisfecho & 12.7 & 100.0 \\
No satisfecho & 0.0 & 100.0 \\
\hline
\end{tabular}

Tabla 4: Complicaciones en los pacientes operados por cirugía percutánea durante los 24 meses de seguimiento.

\begin{tabular}{|lrc|}
\hline Complicación & $\%$ & Acumulativo, \% \\
\hline Ninguna & 68.3 & 68.3 \\
Hallux residual & 12.7 & 12.7 \\
Dolor residual por seis meses & 11.1 & 23.8 \\
Infección superficial & 3.2 & 27.0 \\
Eritema & 1.6 & 28.6 \\
Metatarsalgia residual & 1.6 & 30.2 \\
Hiperqueratosis plantar 2 y 5 dedos & 1.6 & 31.8 \\
\hline
\end{tabular}

general y un buen control del dolor prequirúrgico, con una buena satisfacción personal y cuyos resultados se mantienen en al menos dos años de seguimiento.

\section{Referencias}

1. Secretaría de Salud. Diagnóstico y Tratamiento de hallux valgus. Ciudad de México; 2013

2. Nix S, Smith M, Vicenzino B. Prevalence of hallux valgus in the general population: a systematic review and meta-analysis. J Foot Ankle Res. 2010; 3: 21

3. Canale T, Beaty JH. Pie y tobillo. In: Elsevier, editor. Campbell de cirugía ortopédica. Nashville, Tennesee; 2015. 9344.

4. Maffulli N, Longo UG, Marinozzi A, Denaro V. Hallux valgus: effectiveness and safety of minimally invasive surgery. A systematic review. Br Med Bull. 2011; 97: 149-67.

5. Trnka H, Krenn S, Schuh R. Minimally invasive hallux valgus surgery : a critical review of the evidence. Int Orthop. 2013; 37(9): 1731-5.

6. Van Groningen Bart, Van der Steen MC, Reijman M, Bos J, Hendriks JG. Outcomes in chevron osteotomy for hallux valgus in a large cohort. Foot. 2016; 29: 18-24.

7. Cuellar AA, King HM, Martínez AMC, King MAC, De la Concha BF, et al. Corrección de hallux valgus mediante técnica percutánea. Med Sur. 2011; 18(2): 40-5.

8. Agel J, Beskin JL, Brage M, Guyton GP, Kadel NJ, Saltzman CL, et al. Reliability of the foot function index: a report of the AOFAS outcomes committee. Foot Ankle Int. 2005; 26(11): 962-7.

9. Brogan K, Ed M, Lindisfarne E, Eng M, Akehurst H, Farook U, et al. Minimally invasive and open distal chevron osteotomy for mild to moderate hallux valgus. Am Orthop Foot Ankle Soc. 2016; 37(11): 1197-204.

10. Botezatu I, Marinescu R, Laptoiu D. Minimally invasive percutaneous surgery - recent developments of the foot surgery techniques. J Med Life. 2015; 8: 87-93.

11. Bauer T, De Lavigne C, Biau D, De Prado M, Isham S, Laffenétre O. Percutaneous hallux valgus surgery: a prospective multicenter study of 189 cases. Orthop Clin North Am. 2009; 40(4): 505-14.

12. Magnan B, Bondi M, Mezzari S, Bonetti I, Samaila E. Minimallyı invasive surgery of the forefoot: current concept review. Int J Clin Med. 2013; 4: 11-9. 
13. Bauer T. Percutaneous forefoot surgery. Orthop Traumatol Surg Res. 2014; 100(1): 191-204.

14. De Prado M. Complications in minimally invasive foot surgery Minimal-invasive Fubchirurgie: Komplikationen. Fub und Sprunggelenk. 2013; 11(2): 83-94.

15. Biz C, Fosser M, Dalmau-Pastor M, Corradin M, Rodà MG, Aldegheri $\mathrm{R}$, et al. Functional and radiographic outcomes of hallux valgus correction by mini-invasive surgery with Reverdin-Isham and Akin percutaneous osteotomies: a longitudinal prospective study with a 48-month follow-up. J Orthop Surg Res. 2016; 11: 157-70.

16. Giannini S, Faldini C, Nanni M, Martino A Di, Luciani D, Vannini F. A minimally invasive technique for surgical treatment of hallux valgus: simple, effective, rapid, inexpensive (SERI). Int Orthop. 2013; 37(9): 1805-13.

17. Cuellar AA, De Lavigne C, Guillo S, Jambou S, King-Martinez AC. Técnica híbrida para la corrección de hallux valgus moderado y severo. Tobillo y Pie. 2012; 5(1): 21-8. 